A DYNAMICAL AND CHEMICAL STUDY OF NGC 6302

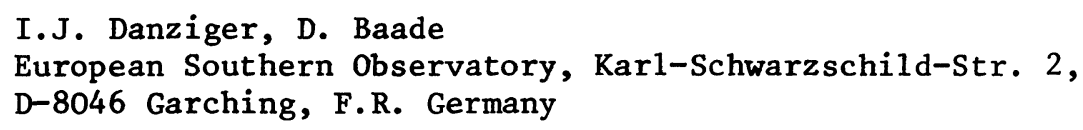

\title{
P.D. Atherton
}

Imperial College of Science \& Technology, The Blackett Laboratory, Prince Consort Road, London SW7 2BZ, U.K.

K. Taylor

Anglo-Australian Observatory, P.0. Box 296, Epping, NSW 2121, Australia
A. Boksenberg
Royal Greenwich Observatory, Hailsham, East Sussex BN27 1RP, U.K.

From five spectrograms obtained at five different positions in the nebula, relative ionic concentrations have been derived with respect to the nucleus. They show that the degree of excitation generally decreases with distance from the nucleus. But there are also areas with locally enhanced or attenuated excitation. Taurus data, a series of twodimensional monochromatic images centered on (OIII) $\lambda$ 5007, have been used to construct a two-dimensional velocity map. It shows a largescale structure similar to the one of direct images with the biconical pattern being at least partly present. Areas of locally lower radial velocity which seem to be inversion symmetrically distributed with respect to the centre, are also distinguished. They do not have pronounced counterparts on direct images. The cavity model suggested by Barral et al. (1982, MNRAS 199, 95) for NGC 6302 and Icke's biconical flow mode1 (1981, Ap. J. 247, 152) are discussed. 